\title{
Aberrant $O$-GlcNAc-modified proteins expressed in primary colorectal cancer
}

\author{
THANONG PHUEAOUAN ${ }^{1}$, PARUNYA CHAIYAWAT ${ }^{1}$, PUKKAVADEE NETSIRISAWAN ${ }^{1}$, \\ DARANEE CHOKCHAICHAMNANKIT ${ }^{2}$, PHAIBUL PUNYARIT ${ }^{3}$, CHANTRAGAN SRISOMSAP $^{2}$, \\ JISNUSON SVASTI ${ }^{1,2}$ and VORARATT CHAMPATTANACHAI ${ }^{1,2}$ \\ ${ }^{1}$ Applied Biological Sciences Program, Chulabhorn Graduate Institute, Bangkok; \\ ${ }^{2}$ Laboratory of Biochemistry, Chulabhorn Research Institute, Laksi, Bangkok; ${ }^{3}$ Department of \\ Clinical Pathology, Army Institute of Pathology, Pramongkutklao Medical Center, Bangkok, Thailand
}

Received June 18, 2013; Accepted August 28, 2013

DOI: $10.3892 /$ or.2013.2794

\begin{abstract}
O$-GlcNAcylation is a post-translational modification of serine and threonine residues which is dynamically regulated by 2 enzymes; $O$-GlcNAc transferase (OGT) and $O$-GlcNAcase (OGA) that catalyze the addition and removal of a single $\mathrm{N}$-acetylglucosamine (GlcNAc) molecule, respectively. This modification is thought to be a nutrient sensor in highly proliferating cells via the hexosamine biosynthesis pathway, a minor branch of glycolysis. Although emerging evidence suggests that $O$-GlcNAc modification is associated with many types of cancer, identification of $O$-GlcNAc-modified proteins and their role in cancer remain unexplored. In the present study, we demonstrated that $O$-GlcNAcylation is increased in primary colorectal cancer tissues, and that this augmentation is associated with an increased expression of OGT levels. Using 2-dimensional $O$-GlcNAc immunoblotting and LC-MS/MS analysis, 16 proteins were successfully identified and 8 proteins showed an increase in $O$-GlcNAcylation, including cytokeratin 18 , heterogeneous nuclear ribonucleoproteins A2/B1 (hnRNP A2/B1), hnRNP H, annexin A2, annexin A7, laminin-binding protein, $\alpha$-tubulin and protein DJ-1. Among these identified proteins, annexin A2 was further confirmed to show overexpression of $O$-GlcNAc in all cancer samples. The results, therefore, indicate that aberrant $O$-GlcNAcylation of proteins is associated with colorectal cancer and that identification of $O$-GlcNAc-modified proteins may provide novel biomarkers of cancer.
\end{abstract}

Correspondence to: Dr Voraratt Champattanachai, Laboratory of Biochemistry, Chulabhorn Research Institute, 54 Kamphaeng Phet 6, Laksi, Bangkok 10210, Thailand

E-mail:voraratt@cri.or.th

Key words: annexin A2, colorectal cancer, hexosamine biosynthesis pathway, $O$-GlcNAcylation

\section{Introduction}

Colorectal cancer is a major medical issue worldwide. It is the third most common cancer in both genders and the second leading cause of cancer-related mortality in the United States (1). In Thailand, although the incidence is low, colorectal cancer is the second most common cancer in men and the third most common cancer in women (2). Colorectal cancer is the most frequent type of cancer among individuals aged 50 years and older, suggesting that the majority of these cancers occur in elder people. However, it has been reported that the incidence rate of colorectal cancer in young individuals (ages 20-49 years) has increased 1.5\%/year in men and 1.6\%/ year in women (3). Therefore, early screening tests are of great important to prevent colorectal cancer and deaths.

The development of colorectal cancer can take years or decades and mainly progresses through the accumulation of many genetic mutations (4). Elevated glucose consumption is a necessary component of carcinogenesis (5). Clinical imaging of primary and metastatic cancers has clearly demonstrated that a common trait of human malignancies including colorectal cancer is elevation in glucose flux compared to normal tissues (6). Changes in the glucose metabolism of tumors lead to the alteration of certain metabolic pathways, including the hexosamine biosynthesis pathway (HBP), a relatively minor branch of glycolysis. A small fraction of glucose enters the HBP and produces uridine diphosphate $N$-acetylglucosamine (UDP-GlcNAc) (7). UDP-GlcNAc is a donor substrate used for classical glycosylation as well as $O$-GlcNAcylation. Unlike classical glycosylation, $O$-GlcNAcylation consists of the attachment of a single $N$-acetylglucosamine (GlcNAc) molecule to serine and threonine residues, which occurs mainly on cytoplasmic, nuclear and mitochondrial proteins. This glycosylation is not static but dynamically regulated by 2 key enzymes, $O$-GlcNAc transferase (OGT) (8) and $O$-GlcNAcase (OGA) (9) which add and remove a sugar molecule from the proteins, respectively.

Abnormal $O$-GlcNAc modification is associated with the pathological status of many diseases including diabetes, neurodegenerative disease, cardiovascular diseases and cancers (10). This modification is thought to act as a nutrient 
sensor in highly proliferating cells including cancer cells. The $O$-GlcNAcylation level was found to be elevated in colorectal and lung cancer (11). Increasing $O$-GlcNAc modification was found to enhance the anchorage-independent growth of colorectal cells in vitro and vice versa for cells observed to be reduced of this modification (11). This modification contributes to the invasion of breast cancer cells in vitro and lung metastasis in vivo (12). Recently, our group demonstrated that $O$-GlcNAc modification was upregulated in breast cancer tissues and decreased $O$-GlcNAcylation was related to inhibition of anchorage-independent growth in vitro (13). We also showed that a number of proteins were selectively modified by $O$-GlcNAc and that these may be novel potential tumor biomarkers in breast cancer (13). However, there is little information concerning $O$-GlcNAc-modified proteins and their roles in other types of cancer. In this study, we explored the expression of $O$-GlcNAcylation, OGT and OGA levels in primary colorectal cancer tissues. Using the combination of proteomic approaches, 2-D IEF/SDS-PAGE, $O$-GlcNAc immunoblotting, followed by LC-MS/MS analysis, we identified a number of proteins associated with aberrant $O$-GlcNAcylation. These results provide the expandable spectrum of $O$-GlcNAcomic in cancer and suggest that aberrant $O$-GlcNAc-modified proteins play a vital role in colorectal cancer.

\section{Materials and methods}

Human colon specimens. Cancerous and adjacent normal parts of 7 colorectal specimens were obtained from Pramongkutklao Hospital (Bangkok, Thailand). Each sample was divided into 2 parts, one for histopathological examination and the other was stored at $-80^{\circ} \mathrm{C}$ until processing. The clinicopathological data for each patient are shown in Table I. The research protocol was approved by the Institutional Review Board of the Royal Thai Army Medical Department, Thailand.

Assessment of O-GlcNAc-modified protein, O-GlcNAc transferase (OGT) and O-GlcNAcase (OGA) levels. Colorectal tissues $(30-50 \mathrm{mg}$ ) were lysed with 1X RIPA supplemented with $1 \%$ protease inhibitor cocktail and $100 \mu \mathrm{M}$ PUGNAc (both from Sigma), an $O$-GlcNAcase inhibitor, homogenized and incubated on ice for $30 \mathrm{~min}$. Soluble proteins $(50 \mu \mathrm{g})$ were separated on $7.5 \%$ SDS-PAGE and transferred to PVDF membranes (Millipore), which were probed with the anti- $O$-GlcNAc antibody CTD110.6, OGT (both from Sigma), and OGA (Abcam). Immunoblots were developed with enhanced chemiluminescence (GE Healthcare), and the signal was recorded on X-ray film and captured by a densitometer (Bio-Rad). Protein loading was compared using gels stained with $0.1 \%$ Coomassie brilliant blue R-250 (CCB) and captured by a densitometer. Densitometric analysis of $O$-GlcNAc proteins was performed on the entire lane of each sample using ImageJ $1.41 \mathrm{v}$ (NIH), and the mean intensity was normalized to the normal groups.

Two-dimensional gel electrophoresis of O-GlcNAc-modified proteins. Colorectal tissues $(30-50 \mathrm{mg})$ were lysed in $2 \mathrm{D}$ lysis buffer [7M urea, 2M thiourea, 4\% CHAPS, 2\% DTT, $2 \%$ ampholine (3-10), $1 \%$ protease inhibitor cocktail (Sigma), and $100 \mu \mathrm{M}$ PUCNAc] homogenized and incubated on ice for
$30 \mathrm{~min}$. Due to limitations of sample amount and to reduce the variation in samples, pooled protein samples were used for $O$-GlcNAc identification. Pooled samples $(1.5 \mathrm{mg})$ were applied by overnight in-gel rehydration of $130-\mathrm{mm}$ nonlinear $\mathrm{pH} 2.0$, IPG strips (GE Healthcare). The strips were then applied to the second dimension and run on 10\% SDS-PAGE, transferred to PVDF membranes (Millipore) and immunoblotted with the anti $O$-GlcNAc antibody. Immunoblots were developed with enhanced chemiluminescence (GE Healthcare) for $1 \mathrm{~min}$ (short exposure time) and 20 min (long exposure time), and the signal was recorded on X-ray film and captured by a densitometer. Immunoblotted membranes were then stripped with stripping buffer (2\% SDS, $62.5 \mathrm{mM}$ Tris-HCl, pH 6.8 and $0.8 \%$ $\beta$-mercaptoethanol) and counter-stained using $0.1 \%$ CBB. Duplicate gels were also stained with $0.1 \%$ CBB for protein identification. $O$-GlcNAc signals of the X-ray film and total protein expression spots stained on stripped membrane were captured by a densitometer. Expression levels of individual $O$-GlcNAc-modified protein spots of the cancer and normal groups were measured by ImageJ 1.41v (NIH). Changes in intensity by $>1.5$-fold were considered to indicate upregulation or downregulation of expression of $O$-GlcNAc levels between cancer and normal groups, respectively.

Protein identification by LC-MS/MS. O-GlcNAc signals obtained from X-ray film were aligned with total protein spots on stripped membranes and CCB gels using Master 2D Platinum 7.0 software (GE Healthcare). Proteins spots from the CCB gels were excised, destained and enzymatically digested by trypsin (Promega Corporation). For protein spots from the PVDF membranes, Zwittergent 3-16 (Merck) at $0.5 \%$ was used for blocking prior to trypsin digestion as described (14). The trypsinized peptides were analyzed by a capillary LC system (Waters) coupled to a Q-TOF mass spectrometer (Micromass) as described previously (13). Briefly, tryptic peptides were injected into a C18 PepMap column (LC Packing) with the gradient of $0.1 \%$ formic acid in $3 \% \mathrm{ACN}$ and $0.1 \%$ formic acid in $97 \%$ ACN. Purified samples $(6 \mu \mathrm{l})$ were injected into the nano-LC. For ESI-Q-TOF analysis, the automatic scan rate was $1.0 \mathrm{sec}$ with an interscan delay of $0.1 \mathrm{sec}$. Parent mass peaks with a range of $400-1600 \mathrm{~m} / \mathrm{z}$ were selected for MS/MS analysis. MS/MS data were processed using MassLynx 4.0 software (Micromass) and converted to PKL files by the ProteinLynx 2.2 software (Waters), which were analyzed using the MASCOT search engine using the NCBInr database (http://www.matrixscience.com). Peptide and fragment mass tolerance were set at 1.2 and $0.6 \mathrm{Da}$, respectively. Proteins with molecular weight and pI consistent with the gel region, having at least one peptide exceeding the score threshold $(\mathrm{P}<0.05)$ were considered as being positively identified.

Validation of O-GlcNAc-modified proteins. A subset of the potential $O$-GlcNAc-modified proteins identified from mass spectrometry was confirmed using the direct immunoprecipitation kit (Pierce Biotechnology, Inc.), performed according to the manufacturer's recommendations. An $O$-GlcNAc antibody (CTD110.6) was used for coupling reaction with the slurry resin (agarose bead) in order to enrich the $O$-GlcNAc proteins from all cancer and normal samples (7 cases). Briefly, proteins $(1 \mathrm{mg})$ in 1X RIPA buffer were incubated with the 
Table I. Clinicopathological data of the colorectal cancer patients.

\begin{tabular}{|c|c|c|c|c|c|c|c|c|}
\hline Case no. & Age (years) & Gender & $\mathrm{T}$ & $\mathrm{N}$ & M & Stage & Grade & Histology \\
\hline 1 & 65 & Female & 3 & 0 & 0 & IIA & II & Adenocarcinoma \\
\hline 2 & 53 & Female & 2 & 0 & 0 & I & II & Adenocarcinoma \\
\hline 3 & 61 & Female & 3 & $2 \mathrm{~A}$ & 0 & IIIB & II & Adenocarcinoma \\
\hline 4 & 78 & Male & 3 & 0 & 0 & IIA & II & Adenocarcinoma \\
\hline 5 & 57 & Female & 2 & 1 & 0 & IIIA & II & Adenocarcinoma \\
\hline 6 & 43 & Female & 3 & 0 & 0 & IIA & II & Adenocarcinoma \\
\hline 7 & 53 & Female & 3 & 1 & 0 & IIIB & II & Adenocarcinoma \\
\hline
\end{tabular}

Three parameters of the TMN classification for colorectal cancer: T, size or direct extent of the tumor; N, degree of spread to axillary lymph nodes; $\mathrm{M}$, presence of metastasis.
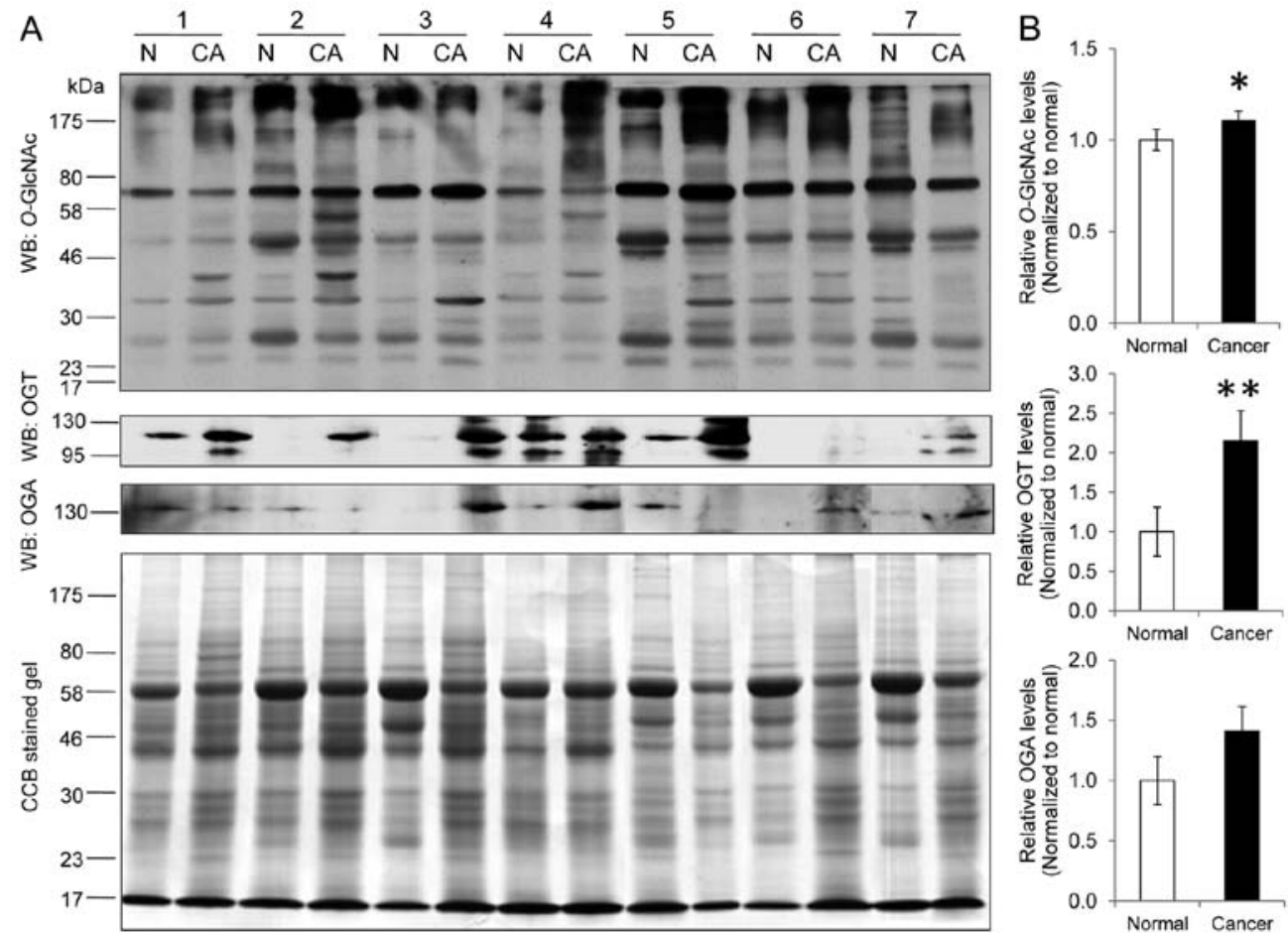

Figure 1. Alteration of $O$-GlcNAcylation, OGT and OGA expression levels in normal (N) and colorectal cancer (CA) tissues. (A) Immunoblots of $O$-GlcNAcylation, OGT, OGA and CCB-stained gel. CCB-stained gel was used as the protein loading. (B) Densitometric analysis of $O$-GlcNAcylation, OGT and OGA levels. $O$-GlcNAcylation, OGT and OGA levels were normalized to each normal group. Data are presented as means \pm SE of 7 samples of colorectal cancer and matched adjacent normal tissue. $\mathrm{N}$, normal; $\mathrm{CA}$, cancer. ${ }^{*} \mathrm{P}<0.05$ vs. normal and ${ }^{* *} \mathrm{P}<0.01$ vs. normal. OGT, $O$-GlcNAc transferase; OGA, $O$-GlcNAcase.

antibody-coupled resin (5 $\mu 1$ of CTD110.6) with gentle shaking overnight at $4^{\circ} \mathrm{C}$. After washing, $100 \mu \mathrm{l}$ of $1 \mathrm{X}$ sampling buffer supplemented with $100 \mathrm{mM}$ DTT was added into the column tube and heated at $95^{\circ} \mathrm{C}$ for $5 \mathrm{~min}$. The eluted samples were centrifuged and loaded in $10 \%$ SDS-PAGE and immunoblotted with the proteins of interest including $O$-GlcNAc, annexin A2 (Abcam), cytokeratin 18 (Chemicon), heat shock cognate protein, and Hsc70 (Abcam). Intensity of the $O$-GlcNAcmodified proteins of the cancer and normal samples was measured by ImageJ 1.41v (NIH).

Data analysis. All data from the densitometric analysis are presented as means \pm standard error (SE). Comparisons were performed using the Student's t-test, and statistically significant differences between groups were defined as $\mathrm{P}<0.05$ and $\mathrm{P}<0.01$.

\section{Results}

Alteration of $O$-GlcNAcylation in colorectal cancer. The expression levels of $O$-GlcNAcylation and $O$-GlcNAc controlling enzymes, OGT and OGA, were examined in 7 cases of colorectal cancer and their adjacent normal tissues (Fig. 1A). $O$-GlcNAc immunoblot analysis revealed a significant increase in the $O$-GlcNAcylation expression level in the cancer group $(1.00 \pm 0.06$ vs. $1.10 \pm 0.05, \mathrm{P}<0.05)$ (Fig. 1B). Notably, 
A

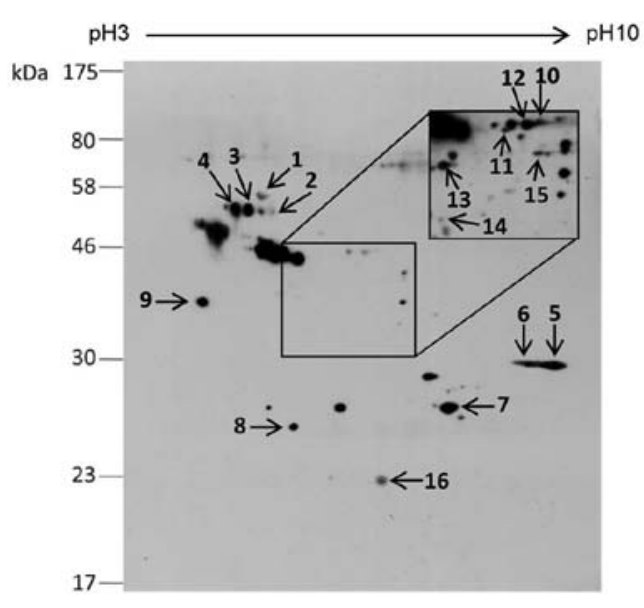

C

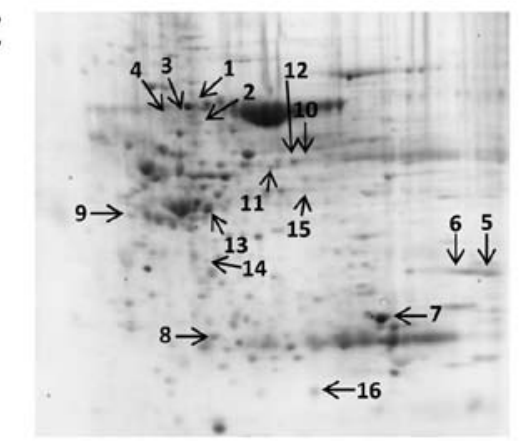

B Cancer

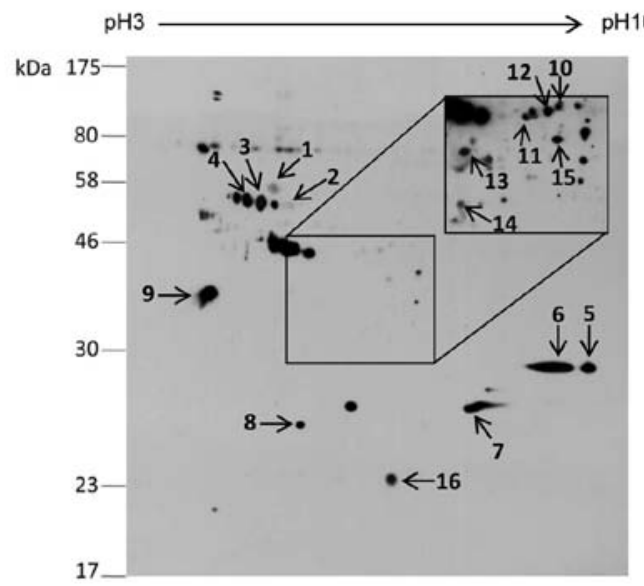

D

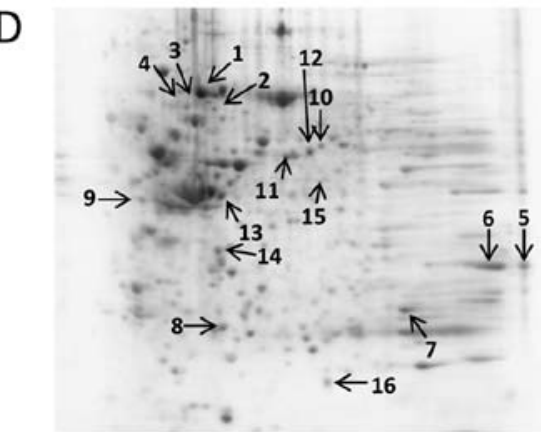

Figure 2. 2-D $O$-GlcNAc immunoblots and protein expression expressed in colorectal tissues. $O$-GlcNAc immunoblots of pooled samples of (A) normal and (B) cancer were separated using 2-D IEF/10\% SDS-PAGE and immunoblotted with an $O$-GlcNAc antibody. Protein expression of (C) normal and (D) cancer was visualized by CCB-stained PVDF membranes. Small boxes are $O$-GlcNAc immunoblots with a longer exposure time (20 min). Arrows with numbers represent the proteins as identified by LC-MS/MS.

several $O$-GlcNAc bands between 17 and $58 \mathrm{kDa}$ were more predominantly presented in tumors compared to those in the normal group. The OGT level was also significantly increased in cancer $(1.00 \pm 0.31$ vs. $2.15 \pm 0.37, \mathrm{P}<0.01)$ whereas the OGA level tended to increase but was not statistically different $(1.00 \pm 0.20$ vs. $1.41 \pm 0.20, \mathrm{P}=0.13)$ in comparison to the normal groups, respectively (Fig. 1B).

Comparison of O-GlcNAc-modified proteins between colorectal cancer and normal tissues. Two dimensional gel electrophoresis and $O$-GlcNAc immunoblot analysis were performed to identify the different $O$-GlcNAc-modified proteins in colorectal cancer. The pooled protein samples of cancerous and normal tissues were used to reduce the complexity of the samples and other errors. Numerous $O$-GlcNAc spots were present in both the cancer and normal 2-D $O$-GlcNAc immunoblots (Fig. 2). However, only $16 O$-GlcNAc protein spots that matched with CCB protein spots were successfully identified. A list of proteins identified and their $O$-GlcNAc expression levels are summarized in Table II. Among the identified proteins, 8 showed increased $O$-GlcNAc expression in cancer (ratio $>1.5$-fold) including cytokeratin 18, heterogeneous nuclear ribonucleoproteins A2/ B1 (hnRNP A2/B1), hnRNP H, annexins A2 and A7, laminin binding protein, $\alpha$-tubulin and protein DJ-1. In contrast,
8 proteins did not differ in regards to the $O$-GlcNAc expression level between the cancer and normal groups (ratio $<1.5$-fold) including heat shock cognate protein (Hsc70), hnRNP K, glyceraldehyde-3-phosphate dehydrogenase (GAPDH), carbonic anhydrase 1, aldehyde dehydrogenase, NADH dehydrogenase, selenium binding protein 1 and creatine kinase-B. All identified proteins resulted from digestion of a single protein spot, except for spot no. 6 which contained 2 proteins, hnRNP A2/ $\mathrm{B} 1$ and annexin $\mathrm{A} 2$.

Confirmatory studies of $O$-GlcNAc-modified proteins in colorectal cancer. A subset of potential $O$-GlcNAc-modified proteins was confirmed using direct immunoprecipitation (IP) with the $O$-GlcNAc antibody followed by immunoblotting with antibodies against the proteins of interest. The results showed that $O$-GlcNAc-modified proteins tended to be increased at higher levels in all cancer samples except sample 6 which showed lower $O$-GlcNAc levels in cancer compared to the normal groups (Fig. 3). $O$-GlcNAc association of annexin A2 was increased in all cancer samples (7/7). $O$-GlcNAc association of cytokeratin 18 (CK18) was increased in 4 cancer samples (4/7) while sample 4 had slighly increased CK18-O-GlcNAc level in normal samples. However, sample 6 and 7 did not show any $O$-GlcNAc-CK18 band in both the normal and cancer samples. Hsc70 associated with $O$-GlcNAc expression was 
Table II. List of identified $O$-GlcNAc-modified proteins in colorectal tissues.

\begin{tabular}{|c|c|c|c|c|c|c|c|}
\hline Spot ID & Accession no. & Protein name & $\begin{array}{l}\text { Mw/pI } \\
\text { matches }\end{array}$ & Peptide & Score & $\begin{array}{c}\text { O-GlcNAc } \\
\text { level }^{\mathrm{a}}\end{array}$ & $\begin{array}{l}O \text {-GlcNAc } \\
\text { (refs.) }\end{array}$ \\
\hline 1 & gil62896815 & Heat shock cognate proteins & $67938 / 5.32$ & 11 & 192 & $\mathrm{NC}$ & $(13,33,35,36)$ \\
\hline 2 & gil30311 & Cytokeratin 18 & $47305 / 5.27$ & 8 & 125 & UP & $(13,22-24)$ \\
\hline 3 & gil460789 & $\begin{array}{l}\text { Heterogeneous nuclear } \\
\text { ribonucleoprotein } \mathrm{K}\end{array}$ & $51040 / 5.13$ & 3 & 27 & $\mathrm{NC}$ & $(13,33)$ \\
\hline 4 & gil460789 & $\begin{array}{l}\text { Heterogeneous nuclear } \\
\text { ribonucleoprotein } \mathrm{K}\end{array}$ & $51040 / 5.13$ & 3 & 48 & $\mathrm{NC}$ & $(13,33)$ \\
\hline 5 & gil31645 & $\begin{array}{l}\text { Glyceraldehyde-3-phosphate } \\
\text { dehydrogenase }\end{array}$ & $36031 / 8.26$ & 6 & 118 & $\mathrm{NC}$ & $(13,33,35)$ \\
\hline \multirow[t]{2}{*}{6} & gil4504447 & $\begin{array}{l}\text { Heterogeneous nuclear } \\
\text { ribonucleoproteins A2/B1 }\end{array}$ & $35984 / 8.67$ & 2 & 62 & UP & $(13,33)$ \\
\hline & gil18645167 & Annexin A2 & $38552 / 7.57$ & 3 & 54 & UP & (13) \\
\hline 7 & gil4502517 & Carbonic anhydrase 1 & $28852 / 6.59$ & 7 & 124 & $\mathrm{NC}$ & - \\
\hline 8 & gil4758788 & NADH dehydrogenase & $30223 / 6.99$ & 7 & 186 & $\mathrm{NC}$ & - \\
\hline 9 & gil34234 & Laminin binding protein & $31774 / 4.84$ & 6 & 175 & UP & - \\
\hline 10 & gil1263008 & Aldehyde dehydrogenase & $57181 / 6.41$ & 6 & 85 & $\mathrm{NC}$ & - \\
\hline 11 & gil5031753 & $\begin{array}{l}\text { Heterogeneous nuclear } \\
\text { ribonucleoprotein } \mathrm{H}\end{array}$ & $49198 / 5.89$ & 3 & 67 & UP & - \\
\hline 12 & gil16306550 & Selenium-binding protein 1 & $52358 / 5.93$ & 14 & 203 & $\mathrm{NC}$ & - \\
\hline 13 & gil180555 & Creatine kinase-B & $42460 / 5.34$ & 6 & 172 & $\mathrm{NC}$ & - \\
\hline 14 & gil340021 & $\alpha$-Tubulin & $50120 / 4.94$ & 7 & 80 & UP & $(25,26)$ \\
\hline 15 & gil4502111 & Annexin A7 & $50284 / 6.25$ & 6 & 57 & UP & - \\
\hline 16 & gil50513593 & Protein DJ-1 & $19886 / 6.33$ & 9 & 105 & UP & (13) \\
\hline
\end{tabular}

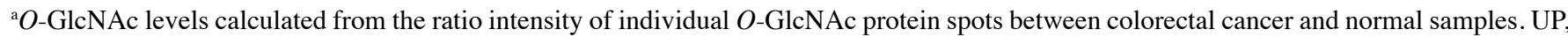
overexpression of $O$-GlcNAc levels in cancer ( $>1.5$-fold); NC, no change in $O$-GlcNAc levels between normal and cancer samples.

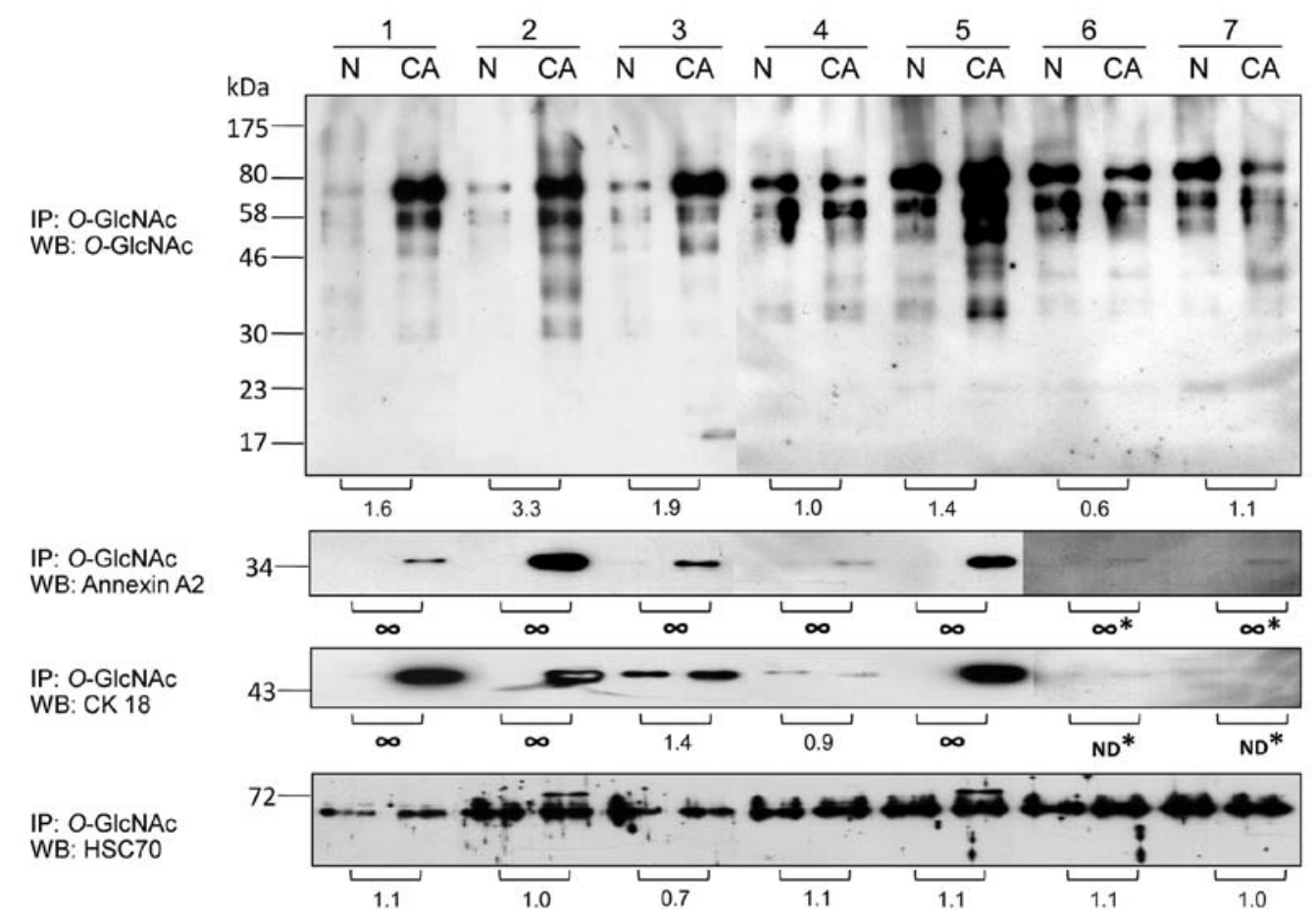

Figure 3. Confirmation of $O$-GlcNAcylation modifications in selected proteins in colorectal cancer. Proteins from the whole cell lysates of individual samples were $O$-GlcNAc-enriched using the $O$-GlcNAc antibody, and the eluted proteins were immunoblotted with selected antibodies including $O$-GlcNAc, annexin A2, CK18 and Hsc70. Numbers below the immunoblots denote the ratio of protein intensity between cancer and normal samples. N, normal; CA, cancer; $\infty$, intensity presented only in cancer; ND, undetectable; *, longer exposure time (20 min). 
slightly altered in all samples, but sample 2 and 5 which had a high $O$-GlcNAc enrichment showed an extra $O$-GlcNAc band of Hsc70 at $72 \mathrm{kDa}$ which was undetectable in all normal samples.

\section{Discussion}

Abnormal $O$-GlcNAcylation is implicated in many metabolic diseases and cancers. In the present study, $O$-GlcNAcylation in primary colorectal tumor tissues was examined in terms of the expression levels of $O$-GlcNAc, OGT and OGA, as well as the identification of $O$-GlcNAc-modified proteins.

$O$-GlcNAc immunoblots showed a significantly different $O$-GlcNAcylated protein pattern between the cancer and normal groups, with several $O$-GlcNAc bands in the molecular weight range from 17 to $58 \mathrm{kDa}$ appearing to increase in cancer (Fig. 1). This increase was associated with an increased expression level of OGT while the OGA level was not significantly different in cancer compared to the normal groups (Fig. 1). This is consistent with a report by Mi and co-workers who demonstrated by immunohistochemistry that the global $O$-GlcNAcylation was significantly elevated in colorectal cancer patients using a different $O$-GlcNAc antibody (RL2) and this elevation was related to an increased OGT level (11). Several lines of evidence have shown that OGT and $O$-GlcNAc levels are increased in many malignant tumors including breast, lung, liver and prostate cancer $(11-13,15,16)$. Recently, it was reported that a metastatic clone of colorectal cancer, SW620, had increased $O$-GlcNAcylation when compared with its primary clone, SW480, and this increase was related to the level of OGA expression in the metastatic clone (17). OGT and OGA may act as key regulatory proteins in cancer, and abnormal addition/removal of $O$-GlcNAcylation in certain proteins, at least, affects protein functions.

In this study, we successfully identified 16 proteins modified by $O$-GlcNAcylation using proteomic approaches including 2-D IEF/SDS-PAGE followed by $O$-GlcNAc immunoblotting and LC-MS/MS, and validated several $O$-GlcNAc-modified proteins using $O$-GlcNAc immunoprecipitation (Table II, Figs. 2 and 3). 2-D proteomic profiles revealed differences in various $O$-GlcNAc-modified proteins between normal and malignant tumors. Eight proteins were associated with increased $O$-GlcNAc in cancer, namely annexins A2 and A7, CK18, $\alpha$-tubulin, hnRNP A2/B1 and H, protein DJ-1 and laminin binding protein.

Annexins are a group of phospholipid-binding proteins in a calcium-dependent manner. They play important roles in cellular signaling such as exocytosis and endoctyosis, cytoskeletal formation, cell matrix interaction, cell division and apoptosis $(18,19)$. We found that $O$-GlcNAc-annexins A2 and A7 were increased in colorectal cancer cases (Fig. 2, Table II). We previously reported that $O$-GlcNAc-annexin A2 was increased in breast cancer tissues (13). It was reported that annexin A2 was significantly increased in primary tumors compared to normal colon and that increased expression of annexin A2 is related to increasing tumor stage (20). Annexin A7 has biological and genetic properties expected of a tumor-suppressor gene. The loss of annexin A7 is associated with distant metastasis in gastric cancer (21). At present, there is no information concerning the glycosylation of annexins.
Abnormal $O$-GlcNAc-annexins may confer benefits to cancer in terms of cellular networking, vesicle transportation, cell matrix formation and metastasis.

Two proteins involved in cell structure, cytokeratin 18 (CK18) and $\alpha$-tubulin, are $O$-GlcNAcylated. CK18 is a type I cytokeratin which forms with its filament partner, cytokeratin $8(\mathrm{CK} 8)$. We found that $O$-GlcNAc-CK18 was increased in colorectal cancer samples similar to that found in our previous study on breast cancer (13). CK18 was also reported to be modified by $O$-GlcNAc, at least in 3 sites (S30/S31/S49) (22). Augmentation of $O$-GlcNAc-CK18 was associated with increased solubility and decreased cellular levels whereas absence of $O$-GlcNAc on CK18 made it more stable (23). $O$-GlcNAcylated $\mathrm{CK} 18$ has been shown to play a protective role in epithelial injury and this glycosylation is required for the recruitment and activation of AKT and protein kinase $\mathrm{C} \theta$ (24). Tubulin is a member of the microtubule family, which is involved in many essential cellular processes, including cell division and intracellular transport. We found that $O$-GlcNAc- $\alpha$-tubulin was increased in cancer. It was reported that $\alpha$-tubulin was identified as an $O$-GlcNAcmodified protein (25). Moreover, it was shown that an increase in $O$-GlcNAc- $\alpha$-tubulin led to reduced heterodimerization and that $O$-GlcNAcylated tubulin did not polymerize into microtubules (26). Aberrant $O$-GlcNAc-CK18 and $O$-GlcNAc-tubulin, therefore, at least may create appropriate structural organization and signaling in the cytoskeletal network of cancer cells. However, the role of this glycosylation on the protein cytoskeleton still requires further study in cancer biology.

The hnRNPs are a group of RNA-binding proteins implicated in a variety of processes in mRNA metabolism including pre-mRNA splicing, mRNA transport and translation $(27,28)$. We found that hnRNP A2/B1 and $\mathrm{H}$ were increased in terms of $O$-GlcNAc modification (Table II). hnRNP A2/B1 interacts with at least 20 other different hnRNPs and RNA in the nucleus which modulate pre-mRNA processing and splicing (29). Fielding et al (30) reported that hnRNP A2/B1 is detected in patients with lung cancer or lung neoplasms suggesting that it is a clinical marker for early lung cancer detection. HnRNP H functions in the splicing of selected target mRNAs such as $\mathrm{Bcl}-\mathrm{x}$, a member of the Bcl-2 family of proteins that are key regulators of apoptosis (31). Although overexpression and subcellular distribution of many hnRNPs have been described in colorectal cancer, the pathogenesis of cancer linked with hnRNPs is unclear (32). To date, the regulation of hnRNPs is poorly understood; however, phosphorylation is thought to be a regulator of the interaction between nucleic acids and protein partners. Wang et al (33) reported that 9 members of the hnRNP family including hnRNP K, U, R, U, A2/B1, A1, C1/C2, H3 and $\mathrm{A} / \mathrm{B}$, are likely to be modified by $O$-GlcNAc in COS7 cells. We previously reported that $5 \mathrm{hnRNPs}$ including hnRNP U-like protein 2, hnRNP K, hnRNP F, hnRNP M isoform a and hnRNP A2/B1, were all increased in their $O$-GlcNAcylation in breast cancer (13). At present, there is no information concerning the functions of $O$-GlcNAc and the association with the site(s) of this modification of hnRNPs. It is possible that increasing $O$-GlcNAcylation of the hnRNP family may interplay with phosphorylation that regulates alterative mRNA splicing and processing, as well as gene expression of proteins and promotes a phenotype of cancer. 
Protein DJ-1 plays a role as an antioxidant and/or a molecular chaperone. It is also a regulator of the tumor-suppressor protein, PTEN, with stimulatory effects on PI3K-AKT/PKB signaling in lung carcinoma. We previously reported that $O$-GlcNAc-DJ-1 was increased in breast cancer (13). Although DJ-1 was $O$-GlcNAc-altered in colorectal cancer, the effect of this modification in stress response remains unclear.

Laminin binding protein (ribosomal protein SA) is involved in the assembly and/or stability of the $40 \mathrm{~S}$ ribosomal subunit and also functions as a cell surface receptor for laminin which is implicated in a wide variety of biological processes including cell adhesion, differentiation, migration, signaling and metastasis (34). $O$-GlcNAc modification of laminin binding protein was increased in colorectal cancer which is similar to our results found in breast cancer (13).

Another group of proteins associated with slightly altered $O$-GlcNAc in colorectal cancer includes Hsc70, hnRNP K, GAPDH, carbonic anhydrase 1, creatine kinase-B, selenium binding protein 1, aldehyde dehydrogenase and NADH dehydrogenase. Hsc70, a constitutively expressed protein, is a chaperone protein which plays vital roles in protein folding and degradation. We found that a major band of $O$-GlcNAc-Hsc70 ( 70 kDa) was similar between normal and cancer groups but a minor band at $72 \mathrm{kDa}$ with high $O$-GlcNAc enrichment was found in cancer samples. Our group and others previously reported that Hsc70 was modified/associated with $O$-GlcNAc $(13,33,35,36)$. GAPDH is a glycolytic enzyme, which breaks down glucose to provide energy. Our and other groups have reported that GAPDH was modified by $O$-GlcNAc $(13,33,35)$. Carbonic anhydrase (CA) catalyzes the hydration of $\mathrm{CO}_{2}$ to form $\mathrm{HCO}_{3}^{-}$and $\mathrm{H}^{+}$ions which are subsequently contributed to an acidic condition in cells. CA1 is found in the cytoplasm of epithelial cells, specifically expressed at high levels in the colon (37). Overexpression of CAs in cancer cells was found to contribute to cancer invasiveness, and CA inhibitors suppress the invasiveness of renal cancer cells in vitro (38). Creatine kinase (CK) is an enzyme catalyzing the reversible phosphorylation of creatine by ATP, thus playing an important role in energy metabolism. High concentrations of CK may appear in cells with highly cellular activities such as cancer cells (39). Selenium binding protein 1 (SBP1) is a 56-kDa protein which is found in various cell types. The function of SBP1 is unknown. However, it was reported that levels of SBP1 are significantly decreased in various epithelial cancers including colorectal cancer $(40,41)$. Aldehyde dehydrogenase is an important enzyme involved in acetaldehyde metabolism. NADH dehydrogenase is an enzyme located in the inner mitochondrial membrane which catalyzes the transfer of electrons from NADH to coenzyme Q. It is still unknown how $O$-GlcNAc modification occurs in mitochondria.

In conclusion, we demonstrated that $O$-GlcNAcylation was increased in primary colorectal cancer patients (grade II) and that this increase was associated with an increased OGT expression level. Proteomic profiles revealed that $O$-GlcNAc-modified proteins are involved in the cytoskeleton, oxidative response and environment, and RNA metabolism. Some proteins were increasingly modified by $O$-GlcNAc. Among these modified proteins, aberrantly increased $O$-GlcNAc-modified annexin A2 was overexpressed in all cancer samples. Taken together, our findings on the alteration of $O$-GlcNAcylation provide the expanding spectrum of $O$-GlcNAcomics in cancer and suggest that abnormal $O$-GlcNAc-modified proteins, particularly annexin A2, may be novel biomarkers of colorectal cancer. Further studies are needed to clarify the biological roles of $O$-GlcNAc-modified proteins in colorectal cancer in order to understand their regulation and function in cancer.

\section{Acknowledgements}

This study was supported by the Chulabhorn Research Institute and Chulabhorn Graduate Institute, Thailand.

\section{References}

1. Siegel R, Ward E, Brawley O and Jemal A: Cancer statistics, 2011: the impact of eliminating socioeconomic and racial disparities on premature cancer deaths. CA Cancer J Clin 61: 212-236, 2011.

2. Attasara P: Hospital-Based Cancer Registry 2010. National Cancer Institute in Thailand, Bangkok, 2011.

3. Siegel RL, Jemal A and Ward EM: Increase in incidence of colorectal cancer among young men and women in the United States. Cancer Epidemiol Biomarkers Prev 18: 1695-1698, 2009.

4. Gryfe R, Swallow C, Bapat B, Redston M, Gallinger S and Couture J: Molecular biology of colorectal cancer. Curr Probl Cancer 21: 233-300, 1997.

5. Gillies RJ, Robey I and Gatenby RA: Causes and consequences of increased glucose metabolism of cancers. J Nucl Med 49 (Suppl 2): S24-S42, 2008.

6. Chowdhury FU, Shah N, Scarsbrook AF and Bradley KM: (18F) FDG PET/CT imaging of colorectal cancer: a pictorial review. Postgrad Med J 86: 174-182, 2010.

7. Marshall S, Bacote V and Traxinger RR: Discovery of a metabolic pathway mediating glucose-induced desensitization of the glucose transport system. Role of hexosamine biosynthesis in the induction of insulin resistance. J Biol Chem 266: 4706-4712, 1991.

8. Kreppel LK, Blomberg MA and Hart GW: Dynamic glycosylation of nuclear and cytosolic proteins. Cloning and characterization of a unique $O$-GlcNAc transferase with multiple tetratricopeptide repeats. J Biol Chem 272: 9308-9315, 1997.

9. Gao Y, Wells L, Comer FI, Parker GJ and Hart GW: Dynamic $O$-glycosylation of nuclear and cytosolic proteins: cloning and characterization of a neutral,cytosolic $\beta-N$-acetylglucosaminidase from human brain. J Biol Chem 276: 9838-9845, 2001.

10. Hart GW, Slawson C, Ramirez-Correa G and Lagerlof O: Cross talk between O-GlcNAcylation and phosphorylation: roles in signaling, transcription, and chronic disease. Annu Rev Biochem 80: 825-858, 2011.

11. $\mathrm{Mi} \mathrm{W}, \mathrm{Gu} \mathrm{Y}, \mathrm{Han} \mathrm{C}$, et al: O-GlcNAcylation is a novel regulator of lung and colon cancer malignancy. Biochim Biophys Acta 1812: 514-519, 2011.

12. Gu Y, Mi W, Ge Y, et al: GlcNAcylation plays an essential role in breast cancer metastasis. Cancer Res 70: 6344-6351, 2010.

13. Champattanachai V, Netsirisawan P, Chaiyawat P, et al: Proteomic analysis and abrogated expression of $O$-GlcNAcylated proteins associated with primary breast cancer. Proteomics 13: 2088-2099, 2013.

14. Pham VC, Henzel WJ and Lill JR: Rapid on-membrane proteolytic cleavage for Edman sequencing and mass spectrometric identification of proteins. Electrophoresis 26: 4243-4251, 2005.

15. Zhu Q, Zhou L, Yang Z, et al: O-GlcNAcylation plays a role in tumor recurrence of hepatocellular carcinoma following liver transplantation. Med Oncol 29: 985-993, 2012.

16. Lynch TP, Ferrer CM, Jackson SR, Shahriari KS, Vosseller K and Reginato MJ: Critical role of $O$-Linked $\beta-N$-acetylglucosamine transferase in prostate cancer invasion, angiogenesis, and metastasis. J Biol Chem 287: 11070-11081, 2012.

17. Yehezkel G, Cohen L, Kliger A, Manor E and Khalaila I: $O$-linked $\beta-N$-acetylglucosaminylation $(O$-GlcNAcylation) in primary and metastatic colorectal cancer clones and effect of $N$-acetyl- $\beta$-D-glucosaminidase silencing on cell phenotype and transcriptome. J Biol Chem 287: 28755-28769, 2012. 
18. Gerke V and Moss SE: Annexins: from structure to function. Physiol Rev 82: 331-371, 2002.

19. Gerke V, Creutz CE and Moss SE: Annexins: linking $\mathrm{Ca}^{2+}$ signalling to membrane dynamics. Nat Rev Mol Cell Biol 6: 449-461, 2005.

20. Duncan R, Carpenter B, Main LC, Telfer C and Murray GI: Characterisation and protein expression profiling of annexins in colorectal cancer. Br J Cancer 98: 426-433, 2008.

21. Hsu PI, Huang MS, Chen HC, et al: The significance of ANXA7 expression and its correlation with poor cellular differentiation and enhanced metastatic potential of gastric cancer. J Surg Oncol 97: 609-614, 2008

22. Ku NO and Omary MB: Identification and mutational analysis of the glycosylation sites of human keratin 18. J Biol Chem 270 11820-11827, 1995.

23. Srikanth B, Vaidya MM and Kalraiya RD: $O$-GlcNAcylation determines the solubility, filament organization, and stability of keratins 8 and 18. J Biol Chem 285: 34062-34071, 2010.

24. Ku NO, Toivola DM, Strnad P and Omary MB: Cytoskeletal keratin glycosylation protects epithelial tissue from injury. Nat Cell Biol 12: 876-885, 2010.

25. Walgren JL, Vincent TS, Schey KL and Buse MG: High glucose and insulin promote $O$-GlcNAc modification of proteins, including $\alpha$-tubulin. Am J Physiol Endocrinol Metab 284: E424-E434, 2003.

26. JiS,Kang JG,Park SY,Lee J,Oh YJ and Cho JW: $O$-GlcNAcylation of tubulin inhibits its polymerization. Amino Acids 40: 809-818, 2011.

27. Krecic AM and Swanson MS: hnRNP complexes: composition, structure, and function. Curr Opin Cell Biol 11: 363-371, 1999.

28. Martinez-Contreras R, Cloutier P, Shkreta L, Fisette JF, Revil T and Chabot B: hnRNP proteins and splicing control. Adv Exp Med Biol 623: 123-147, 2007.

29. Boukakis G, Patrinou-Georgoula M, Lekarakou M, Valavanis C and Guialis A: Deregulated expression of hnRNP A/B proteins in human non-small cell lung cancer: parallel assessment of protein and mRNA levels in paired tumour/non-tumour tissues. BMC Cancer 10: 434, 2010.

30. Fielding P, Turnbull L, Prime W, Walshaw M and Field JK: Heterogeneous nuclear ribonucleoprotein A2/B1 up-regulation in bronchial lavage specimens: a clinical marker of early lung cancer detection. Clin Cancer Res 5: 4048-4052, 1999.
31. Garneau D, Revil T, Fisette JF and Chabot B: Heterogeneous nuclear ribonucleoprotein $\mathrm{F} / \mathrm{H}$ proteins modulate the alternative splicing of the apoptotic mediator Bcl-x. J Biol Chem 280: 22641-22650, 2005.

32. Hope NR and Murray GI: The expression profile of RNA-binding proteins in primary and metastatic colorectal cancer: relationship of heterogeneous nuclear ribonucleoproteins with prognosis. Hum Pathol 42: 393-402, 2011.

33. Wang Z, Pandey A and Hart GW: Dynamic interplay between $O$-linked $N$-acetylglucosaminylation and glycogen synthase kinase-3-dependent phosphorylation. Mol Cell Proteomics 6: 1365-1379, 2007

34. Gauczynski S, Peyrin JM, Haïk S, et al: The 37-kDa/67-kDa laminin receptor acts as the cell-surface receptor for the cellular prion protein. EMBO J 20: 5863-5875, 2001.

35. Wells L, Vosseller K, Cole RN, Cronshaw JM, Matunis MJ and Hart GW: Mapping sites of $O$-GlcNAc modification using affinity tags for serine and threonine post-translational modifications. Mol Cell Proteomics 1: 791-804, 2002

36. Lefebvre T, Cieniewski C, Lemoine J, et al: Identification of $\mathrm{N}$-acetyl-D-glucosamine-specific lectins from rat liver cytosolic and nuclear compartments as heat-shock proteins. Biochem J 360: 179-188, 2001.

37. Drummond FJ, Sowden J, Morrison K and Edwards YH: Colon carbonic anhydrase 1: transactivation of gene expression by the homeodomain protein Cdx2. FEBS Lett 423: 218-222, 1998.

38. Parkkila S, Rajaniemi H, Parkkila AK, et al: Carbonic anhydrase inhibitor suppresses invasion of renal cancer cells in vitro. Proc Natl Acad Sci USA 97: 2220-2224, 2000.

39. Zarghami N, Giai M, Yu H, et al: Creatine kinase BB isoenzyme levels in tumour cytosols and survival of breast cancer patients. Br J Cancer 73: 386-390, 1996.

40. Li T, Yang W, Li M, et al: Expression of selenium-binding protein 1 characterizes intestinal cell maturation and predicts survival for patients with colorectal cancer. Mol Nutr Food Res 52: 1289-1299, 2008.

41. Kim H, Kang HJ, You KT, et al: Suppression of human seleniumbinding protein 1 is a late event in colorectal carcinogenesis and is associated with poor survival. Proteomics 6: 3466-3476, 2006. 